\title{
Emergency Severity Index Scores Differ by Health Provider's Gender and Patient's Age
}

\author{
Jacob M. Vigil ${ }^{1, *}$, Joe Alcock ${ }^{1,2}$, Patrick Coulombe ${ }^{1}$ and Chance Strenth ${ }^{1}$ \\ ${ }^{1}$ University of New Mexico, USA, ${ }^{2}$ New Mexico Veteran's Affairs Health Care System, Albuquerque, NM, USA
}

\begin{abstract}
Background: Experimental research shows that laywomen express higher levels of empathy than men to pain suffering behaviors in others and female practitioners interact with and may prescribe different levels of pain medication to patients than male practitioners. Objectives: In this study we conduct a small medical records review to examine the possibility that female emergency department nurses assign lower levels (i.e., more urgent, serious, resource extensive) of emergency severity index (ESI) scores of patients presenting for care in the emergency department than male nurses, raising the possibility that patients receive disparate treatment during triage depending on the gender of medical staff. Methods: The patient-provided pain scores and the examiner-determined ESI scores of forty-eight male, emergency department patients (21-89yrs, $\left.M_{\text {age }}=57.2, S D_{\text {age }}=19.3\right)$ were examined across multiple visits, along with the gender of their triage examiner at each visit of (127 total patient/provider interactions). Results: A cross-classified mixed-effects model to analyze the influence of examiner gender on the triage scores that showed a significant Examiner Gender $x$ Patient Age interaction. Further probing showed that female practitioners ascribed significantly lower ESI scores than male practitioners, although this effect was limited to patients younger than 55 years of age. Conclusion: These findings warrant larger-scale investigations of patient and examiner influences on patient treatment, which are necessary for creating more standardized protocols for reliably assessing emergency-care patients and for reducing health disparities in patient treatment quality.
\end{abstract}

Keywords: Emergency department, emergency severity index, gender differences, health disparities, interpersonal, patient care.

\section{INTRODUCTION}

Women observers have been reported to judge the pain suffering behaviors of others as more severe than male observers [1]. These differences have been demonstrated in the laboratory and may be rooted in sexually dimorphic empathy-related neuroendocrine activity, with women recruiting greater amygdala and associated (e.g., 'mirror') neuronal activation than males on average [2-6]. Additional research suggests that women demonstrate a stronger correspondence between neurocortical and verbal indicators of pain empathizing [7], while males tend to be more critical in their evaluations of others, potentially attenuating neurocortical empathizing responses and/or reporting [8]. In addition to gender differences in pain empathizing, people subjected to experimental pain stimuli are also more likely to demonstrate heightened pain sensitivity when they are processed by a female researcher [9-11], and even minimal procedural interactions with female laboratory personnel (e.g., processing consent forms) and mere exposure to a female's voice during experimental pain tasks can lead to hyperalgesia $[12,13]$.

These findings can be understood from a social-signaling perspective of pain expression and pain empathizing behaviors. According to the social-signaling model, expressions of

\footnotetext{
*Address correspondence to this author at the Department of Psychology, University of New Mexico, University of New Mexico, MSC03 2220, Albuquerque, NM, 87131-1161, USA; Tel: 505-277-0374;

E-mail: vigilJ@unm.edu
}

pain operate as an interchange of reciprocal demonstrations of trustworthiness cues with others, and are especially important between relationship partners [14-16]. Females heuristically demonstrate cues associated with altruism and vulnerability more than men, including higher levels of both pain reactions as well as pain-empathizing behaviors $[1,17-$ 20] Due to increased female responsiveness to displays of pain, the social-signaling perspective predicts that people would heuristically report higher levels of pain suffering to females $[1,12,14,19,21]$.

Despite strong empirical evidence and conceptual rationale for the existence of gender differences in pain- and empathy-related signaling, there is a surprising scarcity of research on how these processes may translate into patient clinical care. One study has found that emergency care patients were more likely to report higher pain scores to female examiners, particularly when the patients were experiencing high levels of pain severity [21]. Likewise, there has been extensive research showing that male and female physicians differ when communicating to patients; female physicians spend more time get, engaged in more positive talk, have longer conversations, provide more preventive care services, and engage in more continuous services with their patients than male physicians on average [22-26]. Gender differences in physician screening rates have been linked to patientreported preferences for female physicians for conditions such as cervical and breast cancer [27, 28]. Other research has found that female physicians were more likely than male physicians to prescribe higher doses of analgesics to under- 
served categories of hypothetical patients such as ethnic minorities and other females [29, 30]. In another study, researchers found that female medical students and residents were more likely to diagnose patients with COPD in a clinical scenario than family physicians; however, because sex differences in treatment decisions were not found among the practicing physicians it could be argued that gender differences in physician-influenced treatment quality disappear after one has gained extensive training and experience [31]. No published research to our knowledge has directly examined how gender differences in pain empathizing may contribute to health disparities in actual emergency patient care.

In this preliminary study we examine whether male and female triage nurses ascribe different levels of emergency severity index (ESI) scores to patients receiving emergency care. These scores represent the most commonly used and standardized system for making operational decisions about emergency-care patients' condition severity, treatment acuity, and institutional resource allocation. Emergency severity index (triage) scores have been shown to predict the amount of resources that are devoted to individuals receiving emergency treatment, as well as the probability of hospital admission and location of admission [32, 33]. Thus, triage scores are a valid predictor of overall treatment quality. In this study, we conduct a small medical records review of patients admitted for treatment in an urban emergency department to examine how ESI scores are related to patient pain levels and whether the gender of the triage medical staff influences the ESI scores that patients receive. The ESI score is an accurate predictor of treatment intensity, it is critically important to understand whether gender introduces health care disparities involving the initial stage of patient assessment in the emergency department. Hence, this study addresses the question of whether the level of treatment that patients receive is influenced by the gender of their intake examiner.

\section{METHODS}

\section{Study Design and Setting}

The data consists of ESI scores and pain intensity scores that were obtained during standard triage assessments in the Emergency Department (ED) at the New Mexico Veterans Affairs (VA) Health Care System (Albuquerque, NM). The data had already been collected as part of normal patient services protocols, and patients provided consent for their information to be used for medical (e.g., diagnostic) purposes upon admission. The study was approved by the University of New Mexico Institutional Review Board and the New Mexico VA Health Care System Research and Development Board. All the patients were veterans and the ESI/triage scores were ascribed by medical examiners (predominantly registered nurses) using a numerical scale (ESI 1-5, from most urgent to least resource intensive); the pain scores were provided by patients using a numerical pain scale (VAS 010 , from no pain to worst pain imaginable).

\section{Selection of Participants}

All emergency care patients received a triage score and a pain score as part of initial triage regardless of presenting complaint. Likewise, patients in this analysis were not limited to any specific set of diagnoses. The medical record re- views consisted of a convenience sample of forty-eight male patients, some of those who were previously identified as frequent users of the ED during a performance improvement review of ED utilization. Patient age ranged from 21-89yrs $\left(M_{\mathrm{age}}=57.2 \mathrm{yrs}, S D_{\mathrm{age}}=19.31\right)$. Each patient was seen from 1 to 24 times on separate visits by one of 29 nurses $(20$ females) across a total number of 130 separate visits/triage assignments ( $73 \%$ of patients had either 1 or 2 visits). Pain scores were not available for three of those visits, yielding a final sample of 127 visits.

\section{Analyses}

In order to examine how examiner gender and patient age predict triage score, a cross-classified random-effects (multilevel) model [34] will be used. We use a random-effects model, as opposed to linear regression, to account for the fact that the same examiners provided several ESI ratings. The cross-classification nature of the design is due to the fact that some of the same patients visited the hospital several times. In other words, each ESI score was made by an examiner with regards to a patient, but both examiners and patients contributed to more than one ESI score. Randomeffects models should be used in lieu of linear regression in those situations, because repeated measures within examiners and patients violate the assumption of independence of observations of linear regression.

In our models, each ESI score is nested under a specific examiner and a specific patient. In this way, we allow for the mean triage score to vary across examiners (some examiners might tend to see more pain in patients than other examiners, regardless of which specific patient they are evaluating) and patients (some patients might lead examiners to attribute them with more pain regardless of the specific examiner, e.g. via unique communication styles). We conducted our analyses in R v3.0.2 [35] using maximum likelihood estimation in the package lme4 v1.0-5 [36].

\section{RESULTS}

In order to examine the proportion of variance in triage scores that is due to either patient effects or examiner effects, we first computed intraclass correlations (ICCs). To do so, we estimated an intercept-only model (i.e., with no covariates), with random intercepts allowed to vary across patients $(n=48)$ and examiners $(n=29)$. This model showed that $15 \%$ of the variance in triage scores was due to differences between patients, and $13 \%$ of the variance in triage scores was due to differences between examiners. Consequently, triage scores depended about as much on which specific patient was seen during the visit as on the specific examiner attributing the triage score for that visit.

Next, we added our predictors of interest in our model. Specifically, we added fixed effects for examiner gender $(0=$ male, $1=$ female $)$, patient age (centered at the mean age of the 48 patients, mean $=57.2$ years), and the interaction between examiner gender and patients age. There was no evidence that the effect of patient age on triage score varied across examiners $\left\{\chi^{2}(2)=1.54, p=.463\right\}$, or that the effect of examiner gender on triage score varied across patients $\left\{\chi^{2}(2)=0.44, p=.803\right\}$. Therefore, only the intercepts are allowed to vary across examiners and patients 
in our final model. Since triage scores could be affected by the level of pain reported by the patient and the number of times a particular patient has sought medical help, we also added fixed effects for pain score and visit number at each visit.

The results are shown in Table 1. The intercept is the expected triage score (3.11) given for a 57-year-old patient by a male examiner, controlling for pain level and visit number. The term Examiner Gender is the expected difference in triage scores given by female vs. male examiners $(-0.25)$ for a 57 -year-old patient, controlling for pain level and visit number. The term Patient Age is the expected decrease in triage score given by a male examiner $(-0.009)$ for each 1-year increases in patients age, controlling for pain level and visit number. The interaction term is the expected difference between male and female examiners in the effect of a 1-year increase in patient age on triage score (0.014), controlling for pain level and visit number. Table 1 also shows that after accounting for patient age and examiners gender, neither patients pain level nor visit number had an effect on triage score.

Table 1. Parameter estimates and significance tests of fixed effects for cross-classified random-effects model predicting triage score from examiner gender and patient age, controlling for pain score and visit number.

\begin{tabular}{|c|c|c|}
\hline Fixed Effect & Estimate (SE) & $\boldsymbol{p}$-value \\
\hline \hline Intercept & $3.1111(.1422)$ & $<.001$ \\
\hline Examiner Gender & $-0.2535(.1423)$ & .075 \\
\hline Patient Age & $-0.0092(.0052)$ & .077 \\
\hline Examiner Gender * Patient Age & $0.0139(.0066)$ & .034 \\
\hline Patient Pain Score & $0.0286(.0178)$ & .108 \\
\hline Visit Number & $-0.0165(.0153)$ & .281 \\
\hline
\end{tabular}

Note. Examiner gender coded $0=$ male, $1=$ female. Patient age centered at 57.2 years old, the mean age for all patients. Significance tests based on Wald $\mathrm{z}$ tests.

The interaction between Patients Age and Examiner Gender was significant, meaning that there is a significant difference in how patient age affects triage score for male vs. female examiners. To further investigate this effect, probing of the interaction was done using an online tool [37]. Fig. (1) shows the expected triage score for male and female examiners as a function of patient age for three different patient ages: 21 (the minimum in the sample), 57 (the mean age in the sample), and 89 (the maximum in the sample). Fig. (2) shows how the difference in triage scores given by male vs. female examiners (y axis) varies as a function of patient age (x axis), with 95\% confidence bands around the regression line. As can be seen in Fig. (2), male examiners are expected to attribute higher triage scores than female examiners for younger patients (up to 55 years old, dashed vertical line), whereas no such difference between male and female examiners is expected for older patients (starting at 55 years old, the $95 \%$ confidence intervals include 0 as a possible value for the difference between male and female examiners).

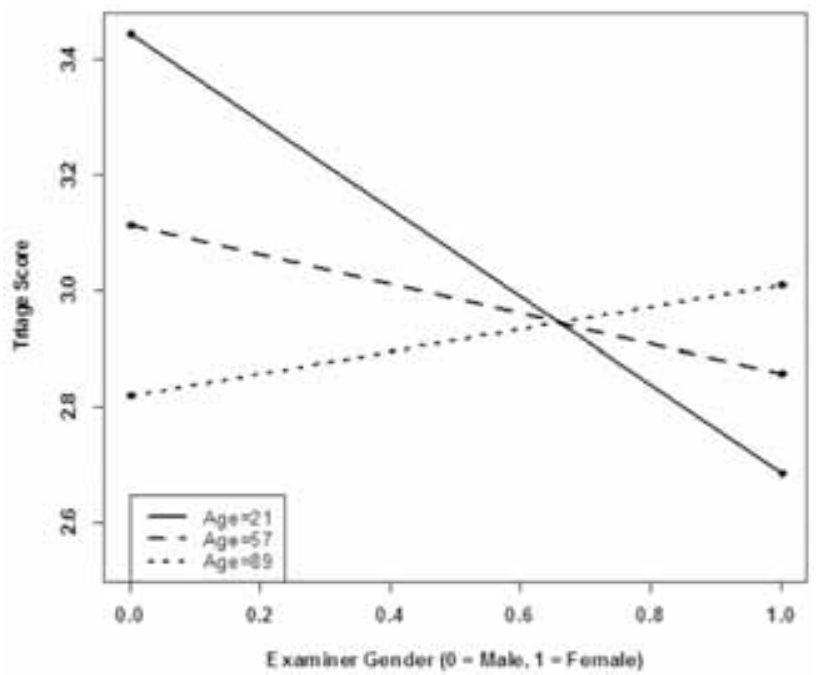

Fig. (1). Examiner gender by patient age interaction.

Note. This figure shows the predicted triage score (y axis) given by male and female examiners (x axis) for three different patient ages: 21 years (the minimum age in the current sample), 57 years (the mean age), and 89 years (the maximum age in the sample).

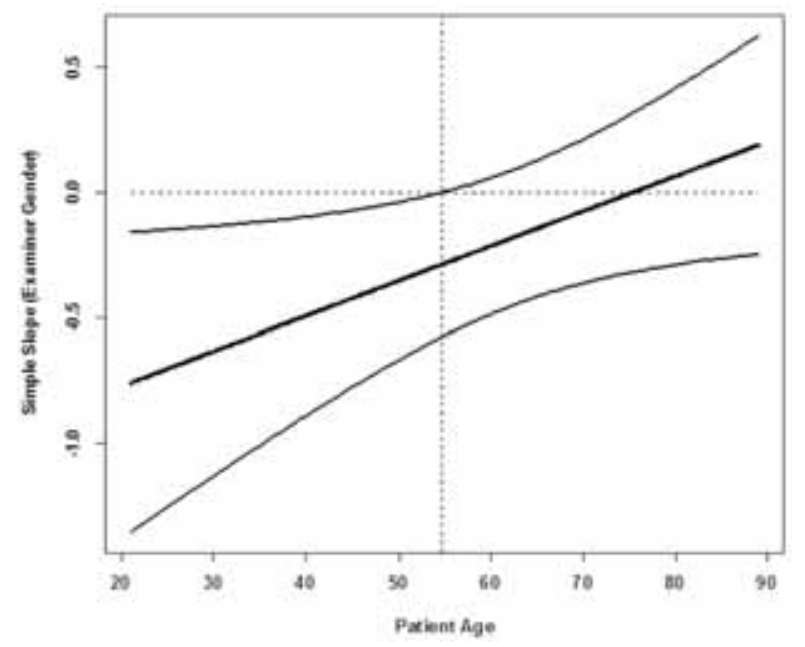

Fig. (2). Confidance bands for the examiner gender effect as a function of patient age.

Note. This figure shows the difference in triage score between female and male examiners (y axis, bold straight diagonal line) as a function of the age of the patient (x axis). The curved lines around the straight line are the lower and upper limits of the $95 \%$ confidence interval of the gender difference at each patient age. The horizontal dashed line is at a male-female difference of 0 , and the vertical dashed line shows where the difference between female and male examiners becomes nonsignificant (to the right side of the vertical dashed line, the value 0 on the y axis falls within the $95 \%$ confidence interval, represented by the two curved lines).

\section{Limitations}

This study has several limitations, including a relatively small sample of patients and nurses at a single, urban emergency department. Another feature that may limit the generalizability of this study is that it was conducted at a Veterans Affairs Medical Center with a population of veterans who represent a predominantly male and older patient population. 
This veteran population, located in the Southwest U.S., is predominantly non-Hispanic White, Hispanic, and Native American, and is underrepresented by African Americans are relatived to many other locations. The data extraction used for this review did not include ethnicity, so the importance of that variable, along with other contextual factors such patient and examiner characteristics, remain to be determined.

\section{DISCUSSION}

This study shows for the first time that the gender of triage examiners (nurses) in the ED interacts with patient age to influence the ESI category assigned to patients presenting to an urban Veteran's Affairs Emergency Department, and ESI/triage scores have been shown to be excellent predictors of hospital admission and death [33]. These scores also likely to predict treatment intensity and may affect the overall treatment quality that patients receive in the emergency department. The present findings also support previous research showing sex differences in diagnosis by female medical personnel; [31] however; this is the first time that these sex differences have been found with medical nurses and the first time that pain empathy of medical personnel has been linked to quality of care. We found a significant interaction between patient age and examiner gender in this study. In general, male and female practitioners diverged in their assignments of triage ESI depending on patient age. Female ED nurses were more likely to ascribe lower ESI scores (more urgent, resource extensive) to younger patients compared to male ED nurses. In general, disparities in triage score diminished with older patients and older patients were less likely to receive disparate ESI scores depending on the gender of their triage examiner than younger patients. Moreover, patient pain did not have an effect on ESI scores after accounting for patient age and gender examiner, and thus it is possible that patient-reported pain levels has a lesser role in determining ESI scores than has been previously reported [32].

Two main findings emerged from this study. First, this work suggests that examiner characteristics can be important in medical assessments, and thus may affect the quality of treatment provided. It is unclear whether these findings may be the result of patient or nursing characteristics such as whether the patients were more vociferous and/or demonstrative to the female nurses, or whether the female nurses could have been more impressed by apparent urgency than were the male nurses. Second, if our findings are validated by future research, it is possible that disparities involving patientexaminer interactions may be more likely among younger patients. At this time, we cannot offer a complete explanation of why there exists a trend towards lower ESI scores (higher acuity) for female examiners and younger patients in particular. One possibility is that older patients may use different communicative styles and/or present more complex phenotypes to medical examiners than younger patients thus otherwise obscuring potential group differences in the treatment decisions of male and female health care workers. Older patients tend to be sicker and more often required to be admitted to the hospital, so they would be expected to have lower triage scores at intake. However, it is possible that emergency nurses may pay more attention to objective cues, such as vital sign abnormalities, and less on subjective cues, such as facial expression in older patients, potentially explaining reduced disparities based on examiner gender. The contrary hypothesis is also possible, however, and it may be that younger patients express more explicit symptom-related behaviors (e.g., facial expressions of pain), and gender differences in emergency nurses decision-making is more closely linked to these factors than to objective cues, per se. It is notable that previous studies have also shown significantly higher pain scores in patients who are younger, but unlike other studies, $[39,40]$ we did not find an association between higher pain scores in patients who have multiple ED visits. It therefore remains possible that the gender differences that we observed occur mostly in certain patient subgroups that tend to report/express higher levels of pain.

\section{Limitations}

The predictive validity of these hypotheses is of course contingent on larger studies among more representative samples of healthcare patients, and thus on investigations that can circumvent the obvious and notable limitations of the current study. The small sample size of patients examined in this medical record review was undoubtingly confounded by innumerable relevant medical, social, and contextual factors that may moderate and even mediate the modulating influence of examiner characteristics (nurse background and life experiences) on triage judgments and other healthcare decisions. Large database studies are needed to determine the variables which are most important in generating ESI scores, including basic demographics and other patient and provider characteristics.

\section{CONCLUSION}

The findings of the present study suggest that health care provider characteristics, such as gender, may influence the course of patient treatment in the emergency department and in other clinical settings. The gender of health practitioners is a ubiquitous contextual factor during all health-provider/ patient interactions, may be a potential source of health disparities in the assessment of patients' needs and thus the overall treatment quality they are likely to receive. The findings also have more basic implications for reliably measuring and interpreting patient health condition, momentarily, across time-points, and across patient populations and health care institutions. The current findings may also elucidate health provider-level factors that implicitly operate alongside other technical factors such as analytical/probabilistic decision-making strategies [38] to influence a broad array of outcomes (e.g., screening, diagnostic effort, continued care) that contribute to potential health disparities in patient treatment quality across a wide-variety of clinical settings. Further research investigating how characteristics of health providers and patients interact is therefore necessary for creating more standardized protocols for reliably assessing patients conditions and for implementing uniformed emergency medical care.

\section{SUMMARY}

1) Why is this topic important? Experimental research shows that laywomen express higher levels of empathy than men to pain suffering behaviors in others which may 
translate into health disparities in patient treatment quality.

2) What does this study attempt to show? In this study we conduct a small medical records review to examine the possibility that female emergency department nurses assign lower levels (i.e., more urgent, serious, resource extensive) of emergency severity index (ESI) scores to patients presenting for care in the emergency department than male nurses, raising the possibility that patients receive disparate treatment during triage depending on the gender of medical staff.

3) What are the key findings? A cross-classified mixedeffects model to analyze the influence of examiner gender on the triage scores showed a significant Examiner Gender $x$ Patient Age interaction. Further probing showed that female practitioners ascribed significantly lower ESI scores than male practitioners, although this effect was limited to patients younger than 55 years of age.

4) How is patient care impacted? These findings show that contextual factors associated with the triage examiner's characteristics influence emergency medical care, and more standardized protocols for reliably assessing emergency-care patients and for reducing health disparities in patient treatment quality are needed.

\section{CONFLICT OF INTEREST}

The authors confirm that this article content has no conflict of interest.

\section{ACKNOWLEDGEMENTS}

This research was supported by a pilot grant from the Clinical and Translational Science Center at the University of New Mexico. The authors declare no financial or other conflicts of interest associated with this work.

\section{REFERENCES}

[1] Vigil JM, Coulombe P. Biological sex and audience affects pain intensity and observational coding of other people's pain behaviors. Pain 2011;152: 2125-30.

[2] Derntl B, Finkelmeyer A, Eickhoff S, et al. Multidimensional assessment of empathic abilities: Neural correlates and gender differences. Psychoneuroendocrinology 2010; 35: 67-82.

[3] Schulte-Rüther M, Markowitsch HJ, Shah NJ, Fink GR, Piefke M. Gender differences in brain networks supporting empathy. Neuroimage 2008; 42: 393-403.

[4] Yang CY, Decety J, Lee S, Chen C, Cheng Y. Gender differences in the mu rhythm during empathy for pain: An electroencephalographic study. Brain Res 2009; 1251: 176-84.

[5] Lamm C, Decety J, Singer T. Meta-analytic evidence for common and distinct neural networks associated with directly experienced pain and empathy for pain. Neuroimage 2011; 54: 2492-502

[6] Preis MA, Schmidt-Samoa C, Dechent P, Kroener-Herwig B. The effects of prior pain experience on neural correlates of empathy for pain: An fMRI study. Pain 2013; 154: 411-8.

[7] Han S, Fan Y, Mao L. Gender difference in empathy for pain: An electrophysiological investigation. Brain Res 2008; 1196: 85-93.

[8] Singer T, Seymour B, O’Doherty JP, Stephan KE, Dolan RJ, Frith CD. Empathic neural responses are modulated by the perceived fairness of others. Nature 2006; 439: 466-9.

[9] Kállai I, Barke A, Voss U. The effects of experimenter characteristics on pain reports in women and men. Pain 2004; 112: 142-7.
[10] Gijsbergs K, Nicholson F. Experimental pain thresholds influenced by sex of experimenter. Percept Mot Skills 2005; 101: 803-7.

[11] McClelland LE, McCubbin JA. Social influence and pain response in women and men. J Behav Med 2008; 31: 413-20.

[12] Vigil JM, Rowell LN, Alcock J, Maestes R. Laboratory personnel gender and cold pressor apparatus affect subjective pain reports. Pain Res Manag 2013; In press.

[13] Vigil JM, Torres D, Wolff A, Hughes K. Exposure to virtual social stimuli modulates subjective pain reports. Pain Res Manag 2013; In press.

[14] Vigil JM, Strenth C. No pain, no social gains: A social-signaling perspective of human pain behaviors. World J Anesth 2013; 2: 1-13

[15] Cano A, Williams A. Social interaction in pain: Reinforcing pain behaviors or building intimacy? Pain 2010; 149: 9-11.

[16] Craig KD. The social communication model of pain. Can Psychol 2009; 50: 22-32.

[17] Vigil, JM. Asymmetries in the friendship preferences and social styles of men and women. Hum Nature 2007; 18: 143-61.

[18] Vigil JM. Sex differences in affect behaviors, desired social responses, and accuracy at understanding the social desires of other people. Evol Psychol 2008; 6: 506-22.

[19] Vigil JM. A socio-relational framework of sex differences in the expression of emotion. Behav Brain Sci 2009; 32: 375-90.

[20] Vigil JM. Current states of opinion and future directions on the epidemiology of sex differences in human pain. Pain Res Manag 2011; 16: 317-9

[21] Vigil JM, Alcock J. Tough guys or cry babies? Disentangling the role of examiner gender on patient pain reports. Pain Res Manag 2013; In press.

[22] Roter, DL Hall JA, Aoki Y. Physician gender effects in medical communication: a meta-analytic review. JAMA 2002; 288: 756-64.

[23] Bertakis KD, Helms LJ, Callahan EJ, Azari R, Robbins JA. The influence of gender on physician practice style. Med Care 1995; 33: 407-16.

[24] Bensing JM, van Den Brink-Muinen A, De Bakker DH. Gender difference in practice style: A Dutch study of general practitioners. Med Care 1993; 31: 219-29.

[25] Rotter D Lipkin M K. Sex difference in patients' and physicians' communication during primary care medical visits. Med Care 1991; 29: 1083-93.

[26] Hall JA, Irish JT, Roter DL, Ehrlich CM, Miller LH. Gender in medical encounters: An analysis of physician and patient communication in a primary care setting. Health Psychol 1994; 13: 384-92.

[27] Hall JA, Palmer H, Orav EJ, Hargraves L, Wright EA, Louis TA. Performance quality, gender, and professional role: A study of physicians and nonphysicians in 16 ambulatory care practices. Med Care 1990; 28: 489-501.

[28] Lurie N, Margolis KL, McGovern PG, Mink PJ, Slater JS. Why do patients of female physicians have higher rates of breast and cervical cancer screening? J Gen Intern Med 1997; 12: 34-43.

[29] Weisse CS, Sorum PC, Dominguez RE. The influence of gender and race on physicians' pain management decisions. J Pain 2003; 4: 505-10.

[30] Weisse CS, Sorum PC, Sanders KN, Syat BL. Do gender and race affect decisions about pain management? J Gen Intern Med 2001; 16: 211-7.

[31] Vajer P, SzeIvari A, Voros K, et al. Comparative analysis of diagnostic probability estimates of some common diagnoses among family doctors, medical residents, and medical students reveals negative correlation between age and estimate of chronic obstructive pulmonary diseases (COPD). Med Sci Monit 2010; 16: 109-15.

[32] Tanabe P, Gimbel R, Yarnold PR, Kyriacou DN, Adams JG. Reliability and validity of scores on the Emergency Severity Index version 3. Acad Emerg Med 2004; 11: 59-65.

[33] Tanabe P, Gimbel R, Yarnold PR, Adams JG. The emergency severity index (version 3) 5-level triage system scores predict ED resource Consumption. J Emerg Nurs 2004; 30: 22-9.

[34] Hox, JJ. Multilevel analysis: Techniques and applications. New York: Taylor \& Francis 2010.

[35] R Core Team (2013). R: A language and environment for statistical computing (Version 3.0.2). Retrieved from http://www.rproject.org

[36] Bates D, Maechler M, Bolker B, Walker S. Linear mixed-effects models using Eigen and S4 (Version 1.0-5). Retrieved from http://CRAN.R-project.org/package=lme4 2013 
[37] Preacher KJ, Curran PJ, Bauer DJ. Computational tools for probing interaction effects in multiple linear regression, multilevel modeling, and latent curve analysis. J Educ Behav Stat 2006; 31: 437-48.

[38] Cahan A, Gilon D, Manor O, Paltiel O. Probabilistic reasoning and clinical decision-making: Do doctors overestimate diagnostic probabilities? QJM 2003; 96: 763-9.
[39] Marco CA, Nagel J, Klink E, Baehren D. Factors associated with self-report pain scores among ED patients. Am J Emerg Med 2012; 30: 331-7.

[40] Marco CA, Kanitz W, Jolly M. Pain scores among emergency department (ED) patients: Comparison by ED diagnosis. J Emerg Med 2013; 44: 46-52.

Received: December 10, 2014

Revised: July 02, 2015

Accepted: July 12, 2015

(C) Vigil et al.; Licensee Bentham Open.

This is an open access article licensed under the terms of the Creative Commons Attribution Non-Commercial License (http://creativecommons.org/licenses/by-nc/3.0/) which permits unrestricted, non-commercial use, distribution and reproduction in any medium, provided the work is properly cited. 